increment of STIs prevalence could be explained: a) due to the rapid expansion of the hot spot areas, b) the increased migration of young women from rural to urban, c) engagement of many young girls in many bars and pubs for commercial sex work activity.

Conclusion The overall prevalence of STIs and HIV was reported $6.8 \%$ and $1.5 \%$ respectively. The prevalence of HIV increased from $0.9 \%$ in 2015 to 1.1 in mid-2018 and STIs from $4.5 \%$ to $6.8 \%$ within the same period. Government in collaboration with the higher institutions and other relevant stakeholders need to further consider strengthening the preventive strategies of STIs and HIV among the key population and other vulnerable groups.

Disclosure No significant relationships.

\section{P817 LONGITUDINAL ASSOCIATIONS BETWEEN RECENT INCARCERATION AND STI/HIV RISK: THE ROLE OF PRIOR TRAUMA IN EXACERBATING RISK}

${ }^{1}$ Kailyn Young, ${ }^{1}$ Willem Van Der Mei, ${ }^{1}$ Joy Scheidell, ${ }^{2}$ Typhanye Dyer, ${ }^{3}$ Christopher HucksOrtiz, ${ }^{4}$ Russell Brewer, ${ }^{1}$ Macregga Severe, ${ }^{1}$ Andrea Troxel, ${ }^{5}$ Jay Kaufman, ${ }^{1}$ Maria Khan* ${ }^{1}$ New York University School of Medicine, Population Health, New York, USA; ${ }^{2}$ University of Maryland, Epidemiology and Biostatistics, College Park, USA; ${ }^{3}$ The HIV Prevention Trials Network, Los Angeles, USA; ${ }^{4}$ University of Chicago, Chicago, USA; ${ }^{5}$ McGill University, Epidemiology, Biostatistics and Occupational Health, Montreal, Canada

10.1136/sextrans-2019-sti.863

Background Black men who have sex with men (BMSM) disproportionately report a history of traumatic life events including incarceration. Incarceration, by increasing distress and psychopathology, may increase risk-taking and infection. Pre-incarceration trauma may exacerbate the impact of incarceration on STI/HIV risk among BMSM.

Methods Using data from HIV Prevention Trials Network (HPTN) 061, we used inverse probability of treatment weighted Poisson regression models to estimate risk ratios (RRs) and 95\% confidence intervals (CIs) for associations between recent incarceration and incident STI (gonorrhea, chlamydia, and syphilis) and sexual risk behavior (sex trade defined as selling/buying sex, multiple partnerships, condomless sex) measured six months after incarceration assessment $(n=1189)$. We tested the significance of interaction terms between incarceration and trauma to assess whether associations differed significantly by trauma history (e.g., experiencing a robbery, natural disaster, sexual/physical assault).

Results Approximately 93\% reported at least one traumatic event and $14 \%$ had been recently incarcerated. Incarceration was associated with STI among those with prior trauma (RR: 1.10, 95\% CI: 1.00-1.22) but not among those with no prior trauma (RR: 0.91, 95\% CI: 0.75-1.09); associations differed significantly (interaction term $\mathrm{p}=0.036$ ). Incarceration was linked to increased risk of sex trade involvement among those with prior trauma (RR: 1.08, 95\% CI: 1.00-1.15) and decreased risk among those with no prior trauma (RR: 0.95, 95\% CI: $0.90-1.00$ ) (interaction term $\mathrm{p}=0.002$ ). Incarceration was associated with increased risk of multiple partnerships among those with prior trauma (RR: 1.24; CI: 1.10, 1.40) but not among those with no prior trauma (RR: $0.85,95 \%$ CI: 0.32-2.25), though the RRs were not significantly different (interaction term $\mathrm{p}=0.224$ ). Incarceration was not associated with condomless sex, regardless of prior trauma.
Conclusion BMSM with prior trauma appear to face disproportionate vulnerability to STI/HIV risk after release from incarceration. Trauma-informed STI/HIV care and prevention interventions for BMSM with recent justice involvement are warranted.

Disclosure No significant relationships.

\section{P818 PREVALENCE OF HPV IN TEENAGE HETEROSEXUAL MALES AFTER THE INTRODUCTION OF THE GENDER- NEUTRAL VACCINATION PROGRAM IN AUSTRALIA}

${ }^{1}$ Eric Chow*, ${ }^{2}$ Sepehr Tabrizi, ${ }^{1}$ Christopher Fairley, ${ }^{1}$ Rebecca Wigan, ${ }^{3}$ David Regan ${ }^{4}$ Jane Hocking, ${ }^{2}$ Suzanne Garland, ${ }^{5}$ Julia Brotherton, ${ }^{1}$ Catriona Bradshaw, ${ }^{2}$ Alyssa Cornall, ${ }^{6}$ Anna McNulty, ${ }^{2}$ Steph Atchison, ${ }^{2}$ Dorothy Machalek, ${ }^{7}$ Louise Owen, ${ }^{8}$ Lewis Marshall, ${ }^{9}$ Darren Russell, ' Marcus Chen. 'Alfred Health, Melbourne Sexual Health Centre, Carlton, Australia; 'The Royal Women's Hospital, Centre for Women's Infectious Disease Research, Parkville, Australia; ${ }^{3}$ UNSW Sydney, The Kirby Institute, Kensington, Australia; ${ }^{4}$ University of Melbourne, Melbourne School of Population and Global Health, Parkville, Australia; ${ }^{5}$ VCS Foundation, National HPV Vaccination Program Register, Melbourne, Australia; ${ }^{6}$ Sydney

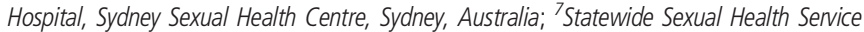
Tasmania, Hobart, Australia; ${ }^{8}$ Fremantle Hospital, South Terrace Clinic, Perth, Australia: ${ }^{9}$ Queensland Health, Cairns Sexual Health Service, Cairns, Australia

\subsection{6/sextrans-2019-sti.864}

Background Australia introduced a school-based human papillomavirus (HPV) vaccination program for females aged 12-13 years in 2007, with a three-year catch-up to age 26; and for boys aged 12-13 from 2013, with a two-year catch-up to age 15. This study aimed to compare the prevalence of penile HPV between teenage heterosexual males in cohorts eligible or non-eligible for the school-based male vaccination program. Methods Between 2014 and 2017, sexually active heterosexual males aged 17-19 were recruited from sexual health centres and community sources across Australia. Males provided a self-collected penile swab for HPV genotypes (Roche Linear Array) and completed a questionnaire. HPV prevalence was compared between males in two periods: 2014-2015 (non-eligible for school-based male vaccination) and 2016-2017 (eligible for school-based male vaccination). Self-reported vaccine doses were confirmed with doses reported to the National HPV Vaccination Program Register.

Results Overall, 152 males were recruited in 2014-2015 and 146 in 2016-2017. Numbers of female sex partners and condom use did not differ between the two periods. Prevalence of quadrivalent vaccine-preventable [4vHPV] genotype $(6,11$, $16,18)$ was low in both periods $(2.6 \%$ in $2014-2015$ versus $0.7 \%$ in 2016-2017; $p=0.37$ ). Compared with males recruited in 2014-2015, males in 2016-2017 had a lower prevalence of: any $37 \mathrm{HPV}$ genotype $(21.7 \%$ versus $11.6 \% ; p=0.02)$; and any 13 high-risk genotype $(15.8 \%$ versus $7.5 \% ; p=0.03)$. Prevalence of low-risk HPV genotypes did not differ between the two periods $(p=0.25)$. Of the males recruited in 20162017, 55\% had received $\geq 1$ vaccine dose.

Conclusion Prevalence of $4 \mathrm{vHPV}$ genotypes among teenage heterosexual males in both cohorts was low, presumably due to herd protection from the female-only vaccination program. The addition of the school-based male vaccination was associated with a lower prevalence of high-risk HPV genotypes other than genotypes 16/18.

Disclosure No significant relationships. 